\title{
The Role of Obstetricians and Neonatologists in Promoting COVID-19 Vaccination: Healthier Mothers and Infants
}

Ricardo Peverini, M.D., Elba Fayard, M.D., Courtney Martin, MD, T. Allen Merritt, MD.

The COVID-19 pandemic has had a profound impact on healthcare systems and pregnancy outcomes. The adverse effects on maternal and neonatal health have not been limited to morbidity and mortality caused directly by SARS-CoV1-19, but also in disruption of prenatal care and fear of attending health care facilities that may have affected maternal well-being and their babies. (1) Rates of stillbirth and preterm birth have changed substantially during pandemic worldwide. (2) A global systematic review found increased maternal mortality and stillbirths, increased ruptured ectopic pregnancies, and maternal stress during the pandemic compared to data prior to the pandemic, including the U.S., Canada, and the UK, as well as in moderate-income countries. These disparities are most significant in low resource settings. (3)

\section{"A global systematic review found increased maternal mortality and stillbirths, increased ruptured ectopic pregnancies, and maternal stress during the pandemic compared to data prior to the pandemic, including the U.S., Canada, and the UK, as well as in moderate-income countries. These disparities are most significant in low resource settings. (3)"}

In the US, as of 3/1/21, 73,600 COVID-19 infections and 80 maternal deaths have occurred in pregnant women. (4) SARS-CoV-2 infection is more severe in pregnant women compared to nonpregnant women with an increased risk of hospitalization, intensive care unit admission, and death. (5) However, despite their increased risk, pregnant and lactating women were not eligible included in the initial COVID-19 vaccine trials. However, about 15,000 incidentally pregnant women received either the Pfizer/BioNTech or Moderna vaccines during the initial clinical trials, and "no red flags" were identified in terms of adverse events. (6)

Although these safety and efficacy trials did not intend to enroll pregnant women because of heightened safety concerns, (7-9) pregnant women (primarily healthcare workers) received the vaccine. These concerns for enrolling pregnant or lactating women were heightened due to the novelty of vaccines utilizing mRNA to deliver SARS-VoV-2 Spike to stimulate the immune system. (10) While infection with wild type SARS-CoV-2 produced antibody responses in mothers with the placental transfer of IgA and IgG to the fetus and newborn, it was unclear whether the mRNA spike protein in novel vaccines would stimulate an identical or similar immunologic response with maternal antibodies transferred to their newborns and via breastmilk.
Recently Gray et al. (11) reported data from 84 pregnant, 31 lactating, and 16 non-pregnant women (mostly healthcare workers) at two medical centers regarding their responses to either Pfizer/BioNTech or Modera mRNA vaccines to deliver SARS-CoV-2 spike antigens to the maternal immune system. Those enrolled were primarily White, non-Hispanic women. Some had reported prior SARS-CoV-2 infection (2 pregnant, 2 lactating, and 1 non-pregnant). The gestational age at vaccination was 23.2 weeks (mean), with 11 women receiving their first dose in the first, 39 during the second, and 34 in the third trimester. Notably, adverse effects during the first dose were minimal, with vaccine-related pyrexia noted in 32 pregnant and $50 \%$ non-pregnant women. Thirteen women delivered during the study (one at 35 weeks) and cord blood was available for only some of the newborns delivered. Maternal IgM, $\lg G$, and $\lg A$ responses to the Spike receptor-binding domain (RBD) and S1 segment of S and S2 of S were measured. The immune response was robust and did not differ amongst pregnant or lactating women. Robust IgM and IgA responses were found with less IgG. In all groups and by two weeks after the second dose, IgG was the dominant induced serum antibody for pregnant, lactating, and non-pregnant women. mRNA vaccination resulted in the induction of antibodies in the sera of vaccinated mothers; however, IgG levels were predominantly induced in breast milk compared to maternal serum.

\section{"In all groups and by two weeks after the second dose, IgG was the dominant induced serum antibody for pregnant, lactating, and non-pregnant women. $m R N A$ vaccination resulted in the induction of antibodies in the sera of vaccinated mothers; however, IgG levels were predominantly induced in breast milk compared to maternal serum."}

Further maternal IgG crossed the placenta to providing immunity to the newborn in $10 / 10$ umbilical cord blood after maternal vaccination.; however, neutralizing antibodies were lower in the cord than the maternal sera. A second dose boost augmented IgG levels in maternal sera, translating to the enhanced transfer of IgG to the neonate through the placenta and via breastmilk. While IgA dominates the antibody transferred from natural COVID-19, it is unknown whether $\operatorname{lgG}$ or $\lg A$ is more important for neonatal protection. These small study results provide strong evidence that COVID-19 mRNA vaccines induce similar humoral immunity in pregnant and lactating women than seen in non-pregnant. (11)

A recent study reported by Pearl et al. also showed robust secretion of SARS-CoV-2 specific IgA and IgG antibodies in breast milk for six weeks after vaccination in 84 lactating subjects. IgA secretion was evident as early as two weeks after vaccination, followed by a spike in IgG after four weeks (a week after the second vaccine). (12) A few other studies have shown similar findings in 
women infected with COVID Antibodies found in the breastmilk of these women showed strong neutralizing effects, suggesting a potential protective effect against infection in the infant. (13)

Vaccination during pregnancy must show evidence of maternal and fetal benefits and not have adverse effects on pregnancy outcomes. Shimabukuro presented data on adverse events among pregnant and non-pregnant women receiving the vaccination and has established a Vaccine Adverse Event Reporting System (VAERS) at the CDC. VAERs serve as an early warning system to detect possible safety issues with US vaccines. (14) Among the 56,567 receiving the Moderna vaccine and 48,196 receiving the Pfizer-BioNTech vaccine, reports of $6,081(6 \%)$ serious adverse events were reported, including headaches and pyrexia chills, pain, fatigue, nausea, dizziness, injection site pain, pain in the extremity, and dyspnea. Among 154 vaccine-associated adverse events among pregnant women with mean maternal age of 33 (range $16-51$ years) with $51 \%$ receiving vaccine during the firsttrimester pregnancy or neonatal specific conditions were reported in $27 \%$, with spontaneous abortion/miscarriage occurring in 29 but at a rate no different than among non-vaccinated pregnant women and three women developed premature rupture of membranes (data through February 16, 2021). Compared to the US population, PPROM affects $2-4 \%$ of the singleton pregnancy population, versus $1.9 \%$ in the VAERS reporting. (15) Most reports $(73 \%)$ reports of vaccine-associated adverse events among pregnant women involved non-pregnancy-specific adverse events (e.g., local and systemic reactions). (16)

\section{"Based on these new findings and other information, the American College of Obstetrics and Gynecologists have revised their recommendations regarding COVID-19 effective March 24, 2021. They now recommend that COVID-19 vaccines should not be withheld from pregnant individuals and COVID-19 should be offered to lactating individuals similar to non-lactating individuals."}

Based on these new findings and other information, the American College of Obstetrics and Gynecologists have revised their recommendations regarding COVID-19 effective March 24, 2021. They now recommend that COVID-19 vaccines should not be withheld from pregnant individuals and COVID-19 should be offered to lactating individuals similar to non-lactating individuals." Further, ACOG recommends a conversation regarding the use of vaccines under an emergency authorization, but such a conversation should not be a barrier to vaccine access on a timely basis." Furthermore, it is imperative women have a conversation with their providers to consider "the risk and potential severity of maternal disease, including the severity of maternal disease, including the effects of disease on the fetus and newborn" with viral activity in their community and the safety of the vaccine for the pregnant patient and the fetus. These conversations or vaccine counseling visits must not be a barrier or requirement to receive the vaccination. The two series of mRNA vaccine injections recommended for mRNA vaccines require that other vaccines such as Ddap and influenza be deferred for 14 days after completing the COVID -19 vaccination period (i.e., five or six weeks after initiation). There is no need to avoid initiation or discontinue breastfeeding in women who receive COVID-19 vaccination for women who choose to breastfeed. Racial and ethnic disparities in health care delivery and outcomes have been reported from COVID-19 epicenters finding increased morbidity and mortality above the general population. In New York City, Latinx women were disproportionately represented among those affected by SARS-CoV-2 infections compared to white women. Latinx women also differed significantly in the borough of residence, insurance type, and household crowding, suggesting that variations in the urban environment and insurance-associated inequities play a more significant role than socioeconomic status alone in the observed disparities related to SARS-CoV-2 infection in an obstetric population. (17)

The need for health equity has been emphasized, especially in communities of color and where inequities have existed in vaccination distribution, especially in communities with Black and Latinx populations. (17) To meet this health inequity recognizing a disproportionate number of COVID-19 infections among African Americans due to structural racism and adverse social determinants of health, Loma Linda University School of Medicine mobilized a "three-tiered approach" by leveraging their relationships with other religious organizations dedicated to confronting issues of bias and promoting health within the Black communities within San Bernardino County, California.

They worked with pastors, coordinated educational webinars about COVID-19 vaccinations, distributed registration materials, and created appointment lists for community members before attending a vaccination clinic. Because attendance at large vaccination sites was disproportionately low among some ethnic groups (Blacks and Latinx), Loma Linda University mobilized health leaders in the Black community, including physicians, pharmacists, and Black faith leaders, to broaden access and receptiveness for vaccination participation. Further, mobile vaccination clinics were held at a primarily Black church parking lot to offer a familiar atmosphere for vaccinations. (16) In addition, researchers at Loma Linda's School of Public health have encouraged finding ways to leverage technologies to get to those highly vulnerable populations for vaccinations.

Issues to be considered when counseling pregnant persons include data from among Latinx communities; vaccine hesitancy has been attributed to social disadvantage, disease misinformation, immigration fears, and healthcare access challenges. Many Latinx community members believed that the threat was "overexaggerated;" therefore, some protective measures were dismissed. These concerns were coupled with fear of unemployment and eviction, inability to self-protect from COVID-19, reluctance to seek health care if uninsured or undocumented, and, among those undocumented, fear of deportation seriously increased their risk of acquiring COVID-19 and the disproportionate risk of hospitalization and death. Within the health care system, social isolation and loneliness, language difficulties, and hospital discharge with insufficient information or clinical follow-up compounded mistrust of public health efforts. Latinx COVID-19 advocates and faith and Hispanic community resiliency offered some resiliency to public health messaging. However, Latinx individuals need more effective public health messaging to reduce testing and vaccination fears and improve vaccine access. (18) The Catholic Diocese has an extensive reach into the Latinx community (particularly undocumented, farmworkers, and migrant workers). In San Bernardino and Riverside counties, Loma Linda faculty collaborated with the Diocese to provide vaccination sites and improve access. Messaging comes from the priests in Spanish supporting vaccination, emphasizing types of public health messaging shown to be most effective (personal communication). 
The Centers for Disease Control and Prevention, the American College of Obstetricians and Gynecologists, and the Society for Maternal-Fetal Medicine, and the American Academy of Pediatrics have each issued guidance supporting offering the COVID-19 vaccine to pregnant women. Combining both Obstetricians' and Neonatologists' efforts coupled with religious organizations that reach minority women will have a greater combined impact to increase vaccinations in pregnant women. Vaccine compliance to protect both mothers and infants should be encouraged now with the added benefit of transferring maternal antibodies to her infants and quite possibly protecting both mothers and their infants from COVID-19 variants. (18)

\section{"We contend that prospective mothers} should be encouraged to carefully consider the benefits of vaccination on their health and the health of their newborn. Breastfeeding should be encouraged now with the added benefit of transferring maternal antibodies to her infants and quite possibly protecting both her and her infant from COVID-19 variants. (17)"

Why are Obstetricians and Neonatologist responses to COVID-19 immunizations so critically important for mothers and infants?

We contend that prospective mothers should be encouraged to carefully consider the benefits of vaccination on their health and the health of their newborn. Breastfeeding should be encouraged now with the added benefit of transferring maternal antibodies to her infants and quite possibly protecting both her and her infant from COVID-19 variants. (19)

1. An essential role for neonatologists and pediatricians is to ask about COVID-19 vaccination status during the evaluation of newborns.

2. Removing barriers to vaccine access, mobilizing communities with leaders, and especially faith leaders with local access using mobile clinics in community settings will increase the likelihood of healthier mothers and their infants.

3. It is the mission of Obstetricians and Neonatologists to promote healthier mothers and infants. It is a moral obligation to protect mothers and their infants from COVID-19 by encouraging vaccination among all women of reproductive age in our society for their well-being and that of their babies.

\section{References:}

1. Burki T. The indirect impact of COVID-19 on women. Lancet Infect Dis. 2020;20(8):904-5. Epub 2020/08/02. doi: 10.1016/ S1473-3099(20)30568-5. PubMed PMID: 32738239; PubMed Central PMCID: PMCPMC7836874.

2. Khalil $A$, von Dadelszen $P$, Draycott $T$, Ugwumadu A, O'Brien $P$, Magee $L$. Change in the Incidence of Stillbirth and Preterm Delivery During the COVID-19 Pandemic. JAMA. 2020. Epub 2020/07/11. doi: 10.1001/jama.2020.12746. PubMed PMID: 32648892; PubMed Central PMCID: PMCPMC7435343.
3. Chmielewska B, Barratt I, Townsend R, Kalafat E, van der Meulen J, Gurol-Urganci I, et al. Effects of the COVID-19 pandemic on maternal and perinatal outcomes: a systematic review and meta-analysis. The Lancet Global Health. 2021. doi: https://doi.org/10.1016/S2214-109X(21)00079-6.

4. DC.COVID Data Tracker 2021 [updated 3/28/2021]. Available from: https://covid.cdc.gov/covid-data-tracker.

5. Zambrano LD, Ellington $S$, Strid $P$, Galang RR, Oduyebo $T$, Tong VT, et al. Update: Characteristics of Symptomatic Women of Reproductive Age with Laboratory-Confirmed SARS-CoV-2 Infection by Pregnancy Status - United States, January 22-October 3, 2020. MMWR Morb Mortal Wkly Rep. 2020;69(44):1641-7. Epub 2020/11/06. doi: 10.15585/ mmwr.mm6944e3. PubMed PMID: 33151921; PubMed Central PMCID: PMCPMC7643892 Journal Editors form for disclosure of potential conflicts of interest. No potential conflicts of interest were disclosed.

6. Merritt T, Goldstein M, Hallman M, Mazela J. Does Maternal COVID-19 Infection Offer Protection to her Newborn? Will Maternal Immunization for COVID-19 Do the Same? Unresolved Questions, Awaiting Answers. Neonatology Today. 2021;16(2):3-6. doi: https://doi.org/10.51362/neonatology. today/2021216236.

7. Beigi RH, Krubiner C, Jamieson DJ, Lyerly $A D$, Hughes $B$, Riley $L$, et al. The need for inclusion of pregnant women in COVID-19 vaccine trials. Vaccine. 2021;39(6):868-70. Epub 2021/01/16. doi: 10.1016/j.vaccine.2020.12.074. PubMed PMID: 33446385; PubMed Central PMCID: PMCPMC7798437 competing financial interests or personal relationships that could have appeared to influence the work reported in this paper.

8. Bianchi DW, Kaeser L, Cernich AN. Involving Pregnant Individuals in Clinical Research on COVID-19 Vaccines. JAMA. 2021;325(11):1041-2. doi: 10.1001/jama.2021.1865.

9. Minkoff $H$, Ecker J. Balancing risks: making decisions for maternal treatment without data on fetal safety. Am J Obstet Gynecol. 2021. Epub 2021/02/05. doi: 10.1016/j. ajog.2021.01.025. PubMed PMID: 33539824.

10. Shimabukuro T. COVID-19 vaccine safety update. Advisory Committee on Immunization Practices (ACIP) 2021 [updated 2/28/20214/2/2021]. Available from: https://www.cdc. gov/vaccines/acip/meetings/downloads/slides-2021-02/2803-01/05-covid-Shimabukuro.pdf.

11. Gray KJ, Bordt EA, Atyeo C, Deriso E, Akinwunmi B, Young $N$, et al. COVID-19 vaccine response in pregnant and lactating women: a cohort study. Am J Obstet Gynecol. 2021. Epub 2021/03/30. doi: 10.1016/j.ajog.2021.03.023. PubMed PMID: 33775692; PubMed Central PMCID: PMCPMC7997025.

12. Perl SH, Uzan-Yulzari A, Klainer H, Asiskovich L, Youngster M, Rinott E, et al. SARS-CoV-2-Specific Antibodies in Breast Milk After COVID-19 Vaccination of Breastfeeding Women. JAMA. 2021. Epub 2021/04/13. doi: 10.1001/ jama.2021.5782. PubMed PMID: 33843975.

13. Pace RM, Williams JE, Jarvinen KM, Belfort $M B$, Pace CDW, Lackey $K A$, et al. Characterization of SARS-CoV-2 RNA, Antibodies, and Neutralizing Capacity in Milk Produced by Women with COVID-19. mBio. 2021;12(1). Epub 2021/02/11. doi: 10.1128/mBio.03192-20. PubMed PMID: 33563823; PubMed Central PMCID: PMCPMC7885115.

14. Kaiser Family Foundation. Greater than COVID 2021 [4/2/2021]. Available from: https://www.greaterthancovid. org.

15. Practice Bulletin No. 160: Premature Rupture of Membranes. Obstet Gynecol. 2016;127(1):e39-e51. Epub 2015/12/24. doi: 10.1097/aog.0000000000001266. PubMed PMID: 26695586.

16. Abdul-Mutakabbir JC, Casey S, Jews V, King A, Simmons K, 
Hogue MD, et al. A three-tiered approach to address barriers to COVID-19 vaccine delivery in the Black community. The Lancet Global Health. 2021. doi: https://doi.org/10.1016/ S2214-109X(21)00099-1.

17. Emeruwa UN, Spiegelman J, Ona S, Kahe K, Miller RS, Fuchs KM, et al. Influence of Race and Ethnicity on Severe Acute Respiratory Syndrome Coronavirus 2 (SARSCoV-2) Infection Rates and Clinical Outcomes in Pregnancy. Obstet Gynecol. 2020;136(5):1040-3. Epub 2020/07/24. doi: 10.1097/aog.0000000000004088. PubMed PMID: 32701761.

18. Cervantes L, Martin M, Frank MG, Farfan JF, Kearns M, Rubio $L A$, et al. Experiences of Latinx Individuals Hospitalized for COVID-19: A Qualitative Study. JAMA Netw Open. 2021;4(3):e210684. Epub 2021/03/12. doi: 10.1001/ jamanetworkopen.2021.0684. PubMed PMID: 33704475; PubMed Central PMCID: PMCPMC7953277.

19. Callaway E. Rare COVID reactions might hold key to variantproof vaccines. Nature. 2021;592(7852):20-1. doi: 10.1038/ d41586-021-00722-8. PubMed PMID: 33762707.

The authors attest that there are no conflicts of interest.
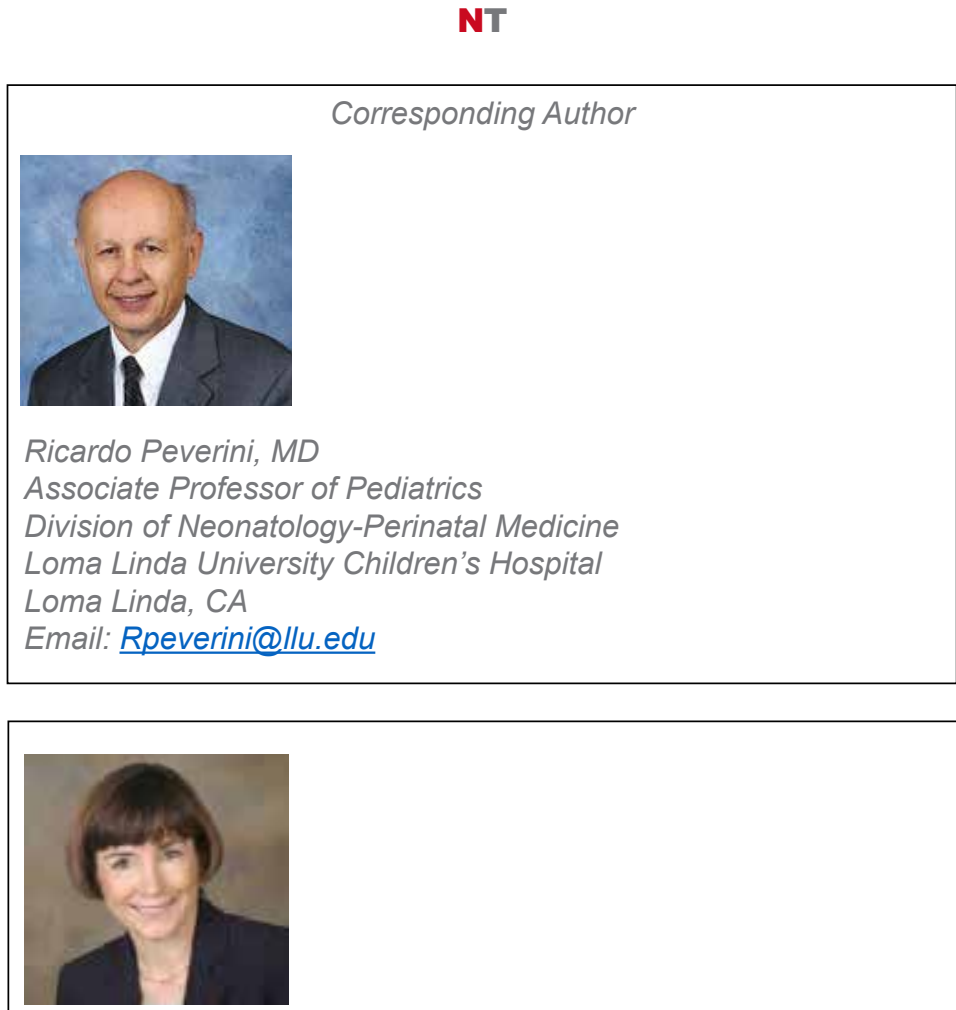

Elba Fayard, $M D$

Professor of Pediatrics

Division Chair

Division of Neonatology-Perinatal Medicine

Loma Linda University Children's Hospital

Loma Linda, CA

Email: Efayard@/lu.edu

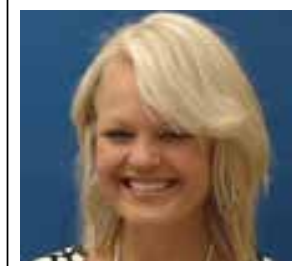

Courtney B. Martin DO FACOG

Chief, Section of General OBGYN

Department of Gynecology and Obstetrics Medical Director, Maternity Services/TCBC

Medical Director, Quality Improvement

Loma Linda University Children's Hospital

Email: Coumartin@llu.edu

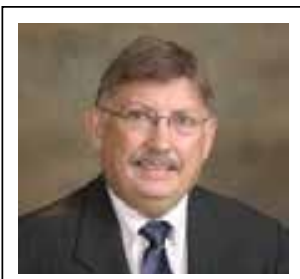

T.Allen Merritt, MD

Professor of Pediatrics

Loma Linda University School of Medicine

Division of Neonatology

Department of Pediatrics

email: T. Allen Merritt < allenmerritt.md@gmail.com>

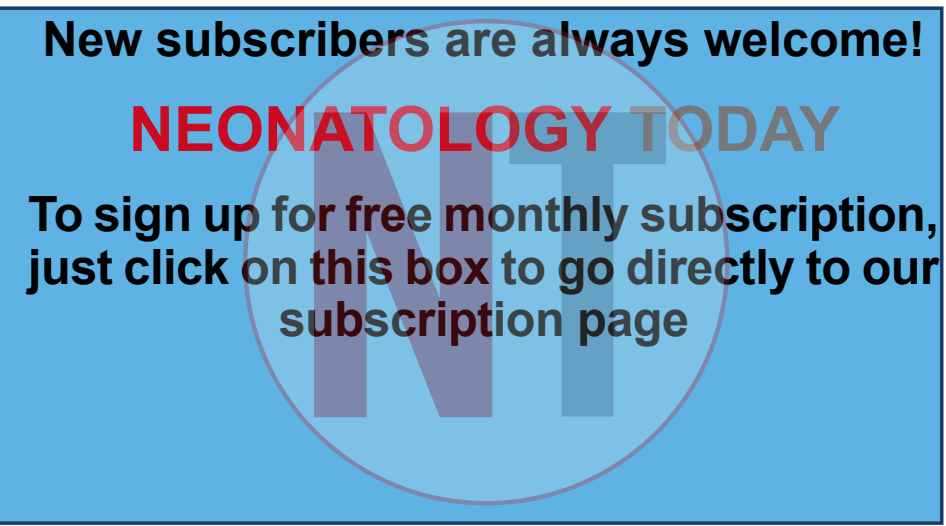

$$
\begin{gathered}
\text { Readers can also follow } \\
\text { NEONATOLOGY } \\
\text { via our Twitter Feed } \\
\text { @NEOTO }
\end{gathered}
$$

